\title{
Efficacy of vildagliptin versus sulfonylureas as add-on therapy to metformin: comparison of results from randomised controlled and observational studies
}

\author{
Bo Ahrén - Chantal Mathieu • Giovanni Bader • \\ Anja Schweizer • James E. Foley
}

Received: 24 January 2014 / Accepted: 7 March 2014 / Published online: 29 March 2014

(C) Springer-Verlag Berlin Heidelberg 2014

\begin{abstract}
Aims/hypothesis Randomised control trials (RCTs) do not always reflect real-life outcomes for glucose-lowering drugs. In this work we compared RCT and real-life data on the efficacy of the dipeptidyl peptidase-IV (DPP-4) inhibitor vildagliptin or sulfonylureas when added to metformin.

Methods Data were pooled from five RCTs examining vildagliptin $(n=2,788)$ and sulfonylureas (glimepiride $[n=1,259]$ or gliclazide $[n=433])$, added to metformin. For real-life conditions, data were extracted from an observational study examining vildagliptin $(n=7,002)$ or sulfonylureas $(n=3,702)$, added to metformin monotherapy. Linear regression analyses were performed between the baseline $\mathrm{HbA}_{1 \mathrm{c}}$ and the change in $\mathrm{HbA}_{1 \mathrm{c}}\left(\Delta \mathrm{HbA}_{1 \mathrm{c}}\right)$ after 24 weeks.

Results Baseline $\mathrm{HbA}_{1 \mathrm{c}}$ correlated to $\Delta \mathrm{HbA}_{1 \mathrm{c}}\left(r^{2}=0.36\right.$, slope $=-0.54$ [95\% CI $-0.55,-0.53 ; p<0.0001])$ for both treatments. With sulfonylureas, the slope of the correlation was steeper in the observational study than in RCTs (interaction coefficient $=-0.327, p<0.001$ ), whereas for vildagliptin, the slope was virtually identical in the observational study and the RCTs (interaction coefficient $=0.024, p=0.175$ ). For any given baseline $\mathrm{HbA}_{1 \mathrm{c}}, \Delta \mathrm{HbA}_{1 \mathrm{c}}$ with sulfonylureas was smaller in real life than in RCTs, whereas $\Delta \mathrm{HbA}_{1 \mathrm{c}}$ with vildagliptin was the same.
\end{abstract}

B. Ahrén

Lund University, Lund, Sweden

C. Mathieu

Catholic University of Leuven, Leuven, Belgium

G. Bader · A. Schweizer

Novartis Pharma AG, Basel, Switzerland

J. E. Foley $(\bowtie)$

Novartis Pharmaceuticals Corporation,

East Hanover, NJ 07936-1080, USA

e-mail: james.foley@novartis.com
Conclusions/interpretations When comparing RCT to reallife data, the decrease in $\mathrm{HbA}_{1 \mathrm{c}}$ from baseline with sulfonylurea treatment is smaller in real life than in RCTs, whereas the reduction with vildagliptin is essentially the same, suggesting that the full power of treatment is retained in real life for vildagliptin but not for sulfonylureas, possibly due to fear of hypoglycaemia.

Keywords DPP-4 inhibitor · GLP-1 · Interventional · Observational $\cdot$ Randomised controlled trial $\cdot$ Sulfonylurea

\begin{tabular}{ll}
\multicolumn{2}{l}{ Abbreviations } \\
$\Delta \mathrm{HbA}_{1 \mathrm{c}}$ & Change from baseline in $\mathrm{HbA}_{1 \mathrm{c}}$ \\
DPP-4 & $\begin{array}{l}\text { Dipeptidyl peptidase-IV } \\
\text { EDGE }\end{array}$ \\
& $\begin{array}{l}\text { Effectiveness of Diabetes control with } \\
\text { vildaGliptin and vildagliptin/mEtformin }\end{array}$ \\
ITT & Intention to treat \\
OAD & Oral antihyperglycaemic drug \\
RCT & Randomised controlled trial
\end{tabular}

\section{Introduction}

Many randomised controlled trials (RCTs) have demonstrated efficacy and safety/tolerability of the dipeptidyl peptidase-IV (DPP-4) inhibitor vildagliptin used as monotherapy and as an add-on to oral antihyperglycaemic drugs (OADs) [1-4]. RCTs meet regulatory and scientific standards, but do not necessarily reflect what happens in real life, and thus do not always provide healthcare professionals with guidance regarding what to expect when prescribing a given drug. It is therefore important to complement the results from RCTs with those from observational trials $[5,6]$. In this study, RCTs of vildagliptin were compared with results of the Effectiveness of Diabetes control with vildaGliptin and vildagliptin/ 
mEtformin (EDGE) trial, which was a non-interventional, non-randomised ( $>45,000$ participants), 1-year observational study comparing vildagliptin with any other OAD added to prior OAD monotherapy in patients with type 2 diabetes and inadequate glycaemic control [7].

The vast majority of patients participating in EDGE were receiving metformin as their initial monotherapy, sulfonylureas were the most common comparator OAD, and many participants had an $\mathrm{HbA}_{1 \mathrm{c}}$ measurement after 24 weeks of treatment as part of their standard clinical care; this allowed the study to compare directly the performance of vildagliptin (87.5\% of EDGE patients in the vildagliptin cohort) vs a sulfonylurea $(72.8 \%$ of EDGE patients in the comparator cohort), both combined with metformin, under real-life conditions, with data obtained from RCTs using the same treatment regimens. Thus, the present post hoc analysis compared the contribution of vildagliptin and sulfonylureas, added to metformin, to the $\mathrm{HbA}_{1 \mathrm{c}}$ reduction at 6 months, in RCTs and real life. To account for differences in $\mathrm{HbA}_{1 \mathrm{c}}$ reductions due to baseline $\mathrm{HbA}_{1 \mathrm{c}}$ levels, $\mathrm{HbA}_{1 \mathrm{c}}$ reductions were analysed relative to the baseline $\mathrm{HbA}_{1 \mathrm{c}}$ levels.

\section{Methods}

Patients and study designs For the RCT population, data were pooled from intention-to-treat (ITT) populations of five clinical trials $[1-3,8,9]$ with 4,480 patients with types 2 diabetes; 2,788 patients received vildagliptin $(50 \mathrm{mg}$ daily $[n=201]$ or $50 \mathrm{mg}$ twice a day $[n=2,587]$ ) plus metformin $\geq 1,500 \mathrm{mg} /$ day and 1,692 patients received a sulfonylurea (glimepiride up to $6 \mathrm{mg} /$ day; $n=1,259$ or gliclazide up to $320 \mathrm{mg} /$ day; $n=433$ ) and metformin $\geq 1,500 \mathrm{mg} /$ day. For the observational population (studied under real-life conditions), data from 10,704 patients $(7,002$ who received vildagliptin $50 \mathrm{mg}$ twice a day added to metformin monotherapy and 3,702 who received a sulfonylurea added to metformin monotherapy) were extracted and summarised from the EDGE study [7]. Dosage information was not collected in the EDGE trial. The RCTs were all randomised, double-blind, controlled clinical trials with a pre-specified week 24 study visit; and in the observational study, oral antihyperglycaemic dual therapy, clinic visits and $\mathrm{HbA}_{1 \mathrm{c}}$ monitoring were solely at the discretion of the physician.

Data analysis Data describing baseline demographic and patient characteristics were summarised for the ITT populations participating in EDGE and RCTs. Baseline and week $24 \mathrm{HbA}_{1 \mathrm{c}}$ levels were measured in each study/population, and linear regression analyses were performed to identify the strongest predictor of treatment response (change from baseline in $\mathrm{HbA}_{1 \mathrm{c}}$ $\left[\Delta \mathrm{HbA}_{1 \mathrm{c}}\right]$ at week 24); baseline $\mathrm{HbA}_{1 \mathrm{c}}$, age, body weight and sex were included in the linear regression model. Additionally,
ANOVA was used to compare the change in $\mathrm{HbA}_{1 \mathrm{c}}$ (adjusted for baseline $\mathrm{HbA}_{1 \mathrm{c}}$ ) between treatments and study conditions.

Ethics and good clinical practice All studies included were conducted in accordance with the Declaration of Helsinki and International Conference on Harmonization/Good Clinical Practice guidelines. The study protocols were approved by an independent ethics committee/institutional review board at each site and all patients provided written informed consent.

\section{Results}

Patient characteristics In all participants the mean $\pm \mathrm{SD}$ age

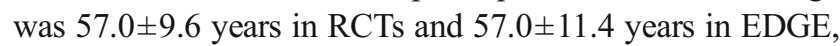
the mean duration of diabetes was $5.1 \pm 4.9$ years in RCTs and $5.4 \pm 5.4$ years in EDGE and about $54 \%$ of patients were male, with little difference between treatment groups or study conditions. Mean baseline $\mathrm{HbA}_{1 \mathrm{c}}$ was higher in both treatment groups in the EDGE population $(8.3 \pm 1.2 \%[67 \pm 13 \mathrm{mmol} / \mathrm{mol}]$ and $8.2 \pm 1.3 \%$ [66 $\pm 14 \mathrm{mmol} / \mathrm{mol}]$ for vildagliptin and sulfonylureas, respectively) than in the RCT population $(7.9 \pm 1.0 \%$ $[63 \pm 11 \mathrm{mmol} / \mathrm{mol}]$ and $7.6 \pm 0.9 \%[60 \pm 10 \mathrm{mmol} / \mathrm{mol}]$ for vildagliptin and sulfonylureas, respectively). Thus, in the RCT population, baseline $\mathrm{HbA}_{1 \mathrm{c}}$ in the vildagliptin group was somewhat higher than the sulfonylurea group.

Predictors of response There was a strong correlation between baseline $\mathrm{HbA}_{1 \mathrm{c}}$ and the response to treatment $\left(\Delta \mathrm{HbA}_{1 \mathrm{c}}\right)$. Assessing the entire data set $(n=12,001), 36 \%$ of the variability in $\Delta \mathrm{HbA}_{1 \mathrm{c}}$ was attributable to baseline $\mathrm{HbA}_{1 \mathrm{c}}\left(r^{2}=0.36\right.$, slope $=-0.54[95 \% \mathrm{CI}-0.55,-0.53$; $p<0.0001])$. In contrast, age, and body weight were nonsignificantly correlated (slopes $<-0.000$ ) and there was very little correlation due to sex (slope $=-0.03, p<0.03$ ).

Figure 1 depicts $\Delta \mathrm{HbA}_{1 \mathrm{c}}$ as a function of baseline $\mathrm{HbA}_{1 \mathrm{c}}$ with sulfonylureas (a) or vildagliptin (b) as add-on to metformin. With sulfonylureas, the slope was $-0.541(95 \%$ CI $-0.56,-0.52 ; p<0.001)$ in RCTs, but was steeper under reallife conditions (in EDGE, interaction coefficient $=-0.327$, $p<0.001$ ). Thus for any given baseline $\mathrm{HbA}_{1 \mathrm{c}}$, in RCTs the $\Delta \mathrm{HbA}_{1 \mathrm{c}}$ with sulfonylurea treatment is greater than in real life. For example, in patients with mean baseline $\mathrm{HbA}_{1 \mathrm{c}}$ of $8.5 \%(69 \mathrm{mmol} / \mathrm{mol})$ the adjusted mean change from baseline $(\mathrm{AM} \Delta)$ in $\mathrm{HbA}_{1 \mathrm{c}}$ in the sulfonylurea treatment group was $-0.9 \%(-10 \mathrm{mmol} / \mathrm{mol})$ in EDGE vs $-1.2 \%(-13 \mathrm{mmol} / \mathrm{mol})$ in RCTs. Furthermore, the difference in $\Delta \mathrm{HbA}_{1 \mathrm{c}}$ reduction between real life and RCTs increased as baseline $\mathrm{HbA}_{1 \mathrm{c}}$ approached normal levels, as illustrated by the shaded area in Fig. 1. In contrast, the regression lines for vildagliptin were nearly superimposable in EDGE and the RCT populations, where the slope of the regression line was -0.55 (95\% CI 

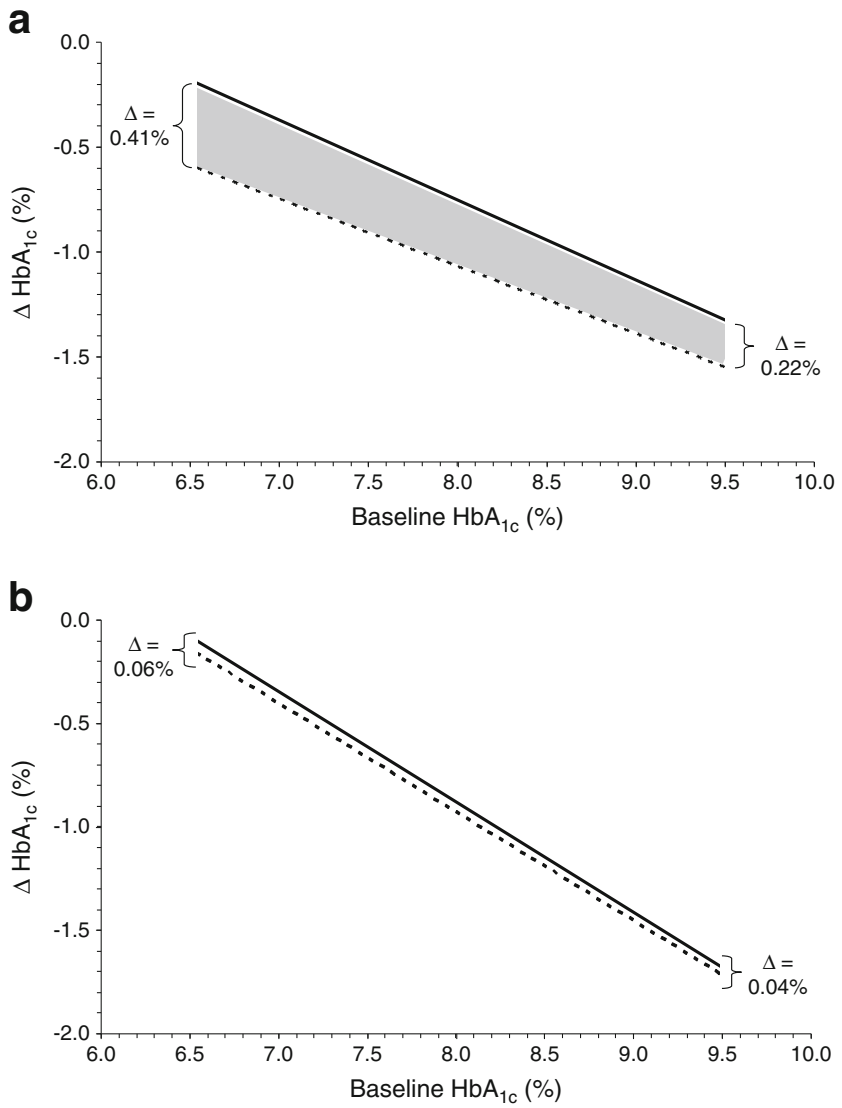

Fig. $1 \Delta \mathrm{HbA}_{1 \mathrm{c}}$ as a function of baseline $\mathrm{HbA}_{1 \mathrm{c}}$ in patients with type 2 diabetes after 24 weeks' treatment with sulfonylurea and metformin (a) or vildagliptin and metformin (b) during an observational study (EDGE, solid line) or RCT (dashed line); interaction coefficient for (a), -0.327 , $p<0.001$; for (b) $0.024, p<0.175$

$-0.56,-0.53 ; p<0.001)$ and there was no interaction with study conditions (RCT vs EDGE, coefficient $=0.024$, $p=0.175)$. In patients with mean baseline $\mathrm{HbA}_{1 \mathrm{c}}$ of $8.5 \%$ $(69 \mathrm{mmol} / \mathrm{mol})$ the adjusted mean $\Delta \mathrm{HbA}_{1 \mathrm{c}}$ in the vildagliptin treatment group was essentially the same in EDGE $(-1.1 \%$; $-12 \mathrm{mmol} / \mathrm{mol})$ and RCTs $(-1.2 \% ;-13 \mathrm{mmol} / \mathrm{mol})$. In the RCTs, body weight increased with sulfonylureas $(1.0 \pm 0.1 \mathrm{~kg}$ from $87.9 \pm 0.4 \mathrm{~kg}, n=1,692)$ and decreased with vildagliptin $(-0.3 \pm 0.1 \mathrm{~kg}$ from $89.2 \pm 0.4 \mathrm{~kg}, n=2,787)$ (both $p<0.001$ ) and in EDGE, body weight reduced with both sulfonylureas $(-0.5 \pm 0.1 \mathrm{~kg}$ from $78.1 \pm 0.3 \mathrm{~kg}, n=2,619)$ and vildagliptin $(-1.4 \pm 0.1 \mathrm{~kg}$ from $81.7 \pm 0.2 \mathrm{~kg}, n=5,045)$ (both $p<0.001$ ).

\section{Discussion}

This post hoc analysis was undertaken to compare the efficacy of sulfonylureas or vildagliptin, used as an add-on to metformin in patients with type 2 diabetes and inadequate glycaemic control, with metformin monotherapy in RCTs vs in a reallife, observational, study. The present analysis confirms the expected relationship between baseline $\mathrm{HbA}_{1 \mathrm{c}}$ and the response to (essentially any) glucose-lowering therapy. While this is usually inferred from subgroup analysis (comparing change from baseline in patients with low vs high baseline $\mathrm{HbA}_{1 \mathrm{c}}$ ), the correlation across a broad range of values is seldom, if ever, reported. Furthermore, the present work examines the relationships between baseline $\mathrm{HbA}_{1 \mathrm{c}}$ and $\Delta$ $\mathrm{HbA}_{1 \mathrm{c}}$ under the two study conditions with the two modes of treatment. The main finding of this work is that, whereas the glycaemic response to vildagliptin in the observational study was entirely consistent with that seen in RCTs, the dependence of the response to sulfonylurea/metformin on baseline $\mathrm{HbA}_{1 \mathrm{c}}$ differed in RCTs and real-life conditions. Hence, the glycaemic response with sulfonylurea treatment was smaller in real life than in RCTs, whereas the glycaemic response with vildagliptin was essentially the same. While the magnitude of the response to sulfonylurea/metformin increased (the change becoming more negative) with increasing baseline $\mathrm{HbA}_{1 \mathrm{c}}$ under both conditions, the blunting of the response became amplified as baseline $\mathrm{HbA}_{1 \mathrm{c}}$ approached normal.

The cause of this blunting of the $\mathrm{HbA}_{1 \mathrm{c}}$ response to sulfonylureas in real life is not clear. However, an important difference between vildagliptin and sulfonylureas is a higher risk for hypoglycaemia with sulfonylureas. Thus, it is tempting to speculate that this blunting is due to fear of the hypoglycaemia that is commonly associated with sulfonylureas [10], and fear of weight gain associated with defensive eating, which may have reduced patient compliance with sulfonylurea therapy, and/or led to a lack of aggressive dose up-titration in observational studies in which no rigid protocol dictates dosing. In contrast, with vildagliptin, the risk for hypoglycaemia (and associated defensive eating) is markedly lower, thereby avoiding the fear of hypoglycaemia and allowing the same patient compliance in real life as in RCTs. The body weight data with sulfonylureas (a small decrease in EDGE and a significant increase in RCTs) is consistent with the above speculation. However, there is no clear explanation for the small differences in weight loss with vildagliptin under the two study conditions, although it should be acknowledged that weight was more systematically determined in the RCTs than in EDGE. The failure to collect dose information in the observational trial is also a limitation of the present comparison.

In summary, this work shows that the lowering of $\mathrm{HbA}_{1 \mathrm{c}}$ with sulfonylurea treatment was diminished in real life relative to RCTs, whereas for vildagliptin, the improvement in glycaemia was the same in RCTs and the observational trial. These data therefore show that the full power of treatment is retained in real life for vildagliptin, whereas sulfonylureas are less efficacious in real life than in RCTs. We suggest that the reduced power of sulfonylureas in real life may be due to fear of hypoglycaemia and the associated weight gain. 
Acknowledgements The authors gratefully acknowledge the members of the steering committees for the two major studies that are included in this analysis, as well the investigators and patients participating in all of the studies included in this analysis.

Funding All studies from which data were compiled for the present analysis were funded by Novartis, as was editorial assistance, provided by Beth Dunning Lower.

Duality of interest BA has received research support, honoraria for speaking engagements and has served on advisory boards for Novartis and has also received honoraria for lecturing or participation in advisory boards from AstraZeneca, GlaxoSmithKline, MSD, Novo Nordisk, Sanofi and Takeda. CM has received honoraria for lectures and/or advisory work from Novo Nordisk, Sanofi, MSD, Eli Lilly, Novartis, Bristol-Myers Squibb, AstraZeneca, Pfizer, Johnson \& Johnson and Mankind; her institution has received research support from Novo Nordisk, Sanofi, MSD, Eli Lilly and Novartis. GB, AS and JEF are employees of Novartis; AS and JEF own shares in Novartis.

Contribution statement All authors had full access to all data and take responsibility for the integrity of the data and accuracy of analyses. All provided input to the analytical approach, interpretation of data, preparation, revision and final approval of the manuscript.

\section{References}

1. Bolli G, Dotta F, Rochotte E, Cohen SE (2008) Efficacy and tolerability of vildagliptin vs. pioglitazone when added to metformin: a 24-week, randomized, double-blind study. Diabetes Obes Metab 10:82-90
2. Bosi E, Camisasca RP, Collober C, Rochotte E, Garber AJ (2007) Effects of vildagliptin on glucose control over 24 weeks in patients with type 2 diabetes inadequately controlled with metformin. Diabetes Care 30:890-895

3. Matthews DR, Dejager S, Ahren B et al (2010) Vildagliptin add-on to metformin produces similar efficacy and reduced hypoglycaemic risk compared with glimepiride, with no weight gain: results from a 2-year study. Diabetes Obes Metab 12:780-789

4. Ahren B, Foley JE, Bosi E (2011) Clinical evidence and mechanistic basis for vildagliptin's action when added to metformin. Diabetes Obes Metab 13:193-203

5. Schwartz D, Lellouch J (2009) Explanatory and pragmatic attitudes in therapeutical trials. J Clin Epidemiol 62:499-505

6. Treweek S, Zwarenstein M (2009) Making trials matter: pragmatic and explanatory trials and the problem of applicability. Trials $10: 37$

7. Mathieu C, Barnett AH, Brath $\mathrm{H}$ et al (2013) Effectiveness and tolerability of second-line therapy with vildagliptin vs. other oral agents in type 2 diabetes: a real-life worldwide observational study (EDGE). Int J Clin Pract 67:947-956

8. Filozof C, Gautier JF (2010) A comparison of efficacy and safety of vildagliptin and gliclazide in combination with metformin in patients with type 2 diabetes inadequately controlled with metformin alone: a 52-week, randomized study. Diabet Med 27:318-326

9. Bosi E, Dotta F, Jia Y, Goodman M (2009) Vildagliptin plus metformin combination therapy provides superior glycaemic control to individual monotherapy in treatment-naive patients with type 2 diabetes mellitus. Diabetes Obes Metab 11:506-515

10. Williams SA, Shi L, Brenneman SK, Johnson JC, Wegner JC, Fonseca V (2012) The burden of hypoglycemia on healthcare utilization, costs, and quality of life among type 2 diabetes mellitus patients. J Diabet Complicat 26:399-406 